\title{
Cloud radiative effect on tropical troposphere to stratosphere transport represented in a large-scale model
}

\author{
Xianglei Huang ${ }^{1}$ and Hui $\mathrm{Su}^{2}$ \\ Received 11 August 2008; revised 26 September 2008; accepted 8 October 2008; published 11 November 2008.
}

[1] GFDL AM2 model simulations are analyzed to assess the simulated radiative effect of tropical tropopause layer (TTL) cirrus on tropical troposphere-to-stratosphere transport (TST). The strongest upward motion in the model's TTL is generally driven by dynamics instead of radiation, occurring in those TTL cloudy regions that overlap with optically thick clouds in the upper troposphere (UT). However, the occurrence frequency of such strong ascent is about one order of magnitude smaller than that of moderate ascent related to the radiative effect of TTL cirrus. The mean upward velocity of moderate ascent in the cloudy regions $(\sim-2.5--3.5 \mathrm{hPa} /$ day $)$ is one order of magnitude larger than that induced by TTL clear-sky radiative heating $(\sim-0.18 \mathrm{hPa} /$ day $)$. This supports the hypothesis that cirrus radiative heating contributes substantially to the average tropical TST rates. The implication for future model-satellite comparisons is discussed. Citation: Huang, X., and H. Su (2008), Cloud radiative effect on tropical troposphere to stratosphere transport represented in a large-scale model, Geophys. Res. Lett., 35, L21806, doi:10.1029/2008GL035673.

\section{Introduction}

[2] The entry of air into the stratosphere can generally be explained by large-scale upwelling in the tropical upper troposphere (UT) [e.g., Brewer, 1949; Holton et al., 1995]. However, the vertical mass transport associated with clear sky radiative heating is found to be too slow to support insitu tracer measurements in the UT and lower stratosphere (LS) [Boering et al., 1994; Andrews et al., 1999; Sherwood and Dessler, 2003]. Different hypotheses have been proposed to explain troposphere-to-stratosphere transport (TST), emphasizing either the gradual ascending towards the stratosphere [e.g., Holton and Gettelman, 2001] or the "fountain"-like deep convective transport [e.g., Sherwood and Dessler, 2001]. The radiative impact of cirrus clouds has been proposed as a possible mechanism for increasing the rate of mass transport from troposphere to stratosphere [Corti et al., 2005, 2006] (hereinafter referred as C05 and C06, respectively).

[3] The tropical tropopause layer (TTL) is the region of the tropical atmosphere that lies between the top of the main cumulus outflow layer $(\sim 12 \mathrm{~km})$ and the thermal tropopause $(\sim 16 \mathrm{~km})$. For a single-layer cloud residing in the TTL, the temperature contrast between the TTL and surface

\footnotetext{
${ }^{1}$ Department of Atmospheric, Oceanic, and Space Sciences, University of Michigan, Ann Arbor, Michigan, USA.

${ }^{2}$ Jet Propulsion Laboratory, California Institute of Technology, Pasadena, California, USA.
}

Copyright 2008 by the American Geophysical Union. 0094-8276/08/2008GL035673 leads to a convergence of longwave flux at the TTL and thus radiatively warms the TTL. For multi-layer clouds, the longwave radiative effect of TTL cirrus is sensitive to the cloud distribution underneath the TTL because the thermal contrast between TTL cirrus and underlying clouds determines the radiative flux divergence in the TTL [Ramaswamy and Ramanathan, 1989; Hartmann et al., 2001; Gettelman et al., 2004]. TTL cirrus overlapped with minimal amount of UT clouds is more likely to radiatively heat the TTL than those overlapped with optically thick clouds in the UT. However, the latter case tends to result from strong upward motion through the entire tropospheric column. In such a case, although the radiative influence of cirrus on upward motion is small or even negative, the dynamically driven upward motion could be strong.

[4] It is useful to examine how TTL transports are simulated in current state-of-the-art general circulation models (GCMs), especially the partitioning between the roles played by dynamics and radiation. Horizontal winds in the TTL are orders of magnitude faster than vertical winds. Thus, the horizontal displacement of an air parcel in the TTL must be taken into account to fully understand the transport. This substantiates the need of a trajectory model in the detailed investigation of cloud radiative impacts on tropical TST pathways. Previous studies using trajectory code have produced useful insights into water vapor dehydration problems and TST pathways [e.g., Dessler and Sherwood, 2000; Jensen and Pfister, 2004; Bonazzola and Haynes, 2004; Fueglistaler et al., 2004; Dessler and Minschwaner, 2007]. The application of trajectory modeling to studying the cirrus radiative impact on tropical TST has not been explored in detail. Thus, it is attractive to use a trajectory modeling approach to examine this issue in a state-of-the-art GCM.

[5] In this study, we use a trajectory model to investigate the radiative impact of TTL cirrus on the tropical TST in the GFDL AM2. The results are instructive to parallel satellite data analysis and facilitate conceptual understanding of the nature of the problem. The models and analysis methods are described in section 2. Section 3 presents the results. The discussion and conclusion are given in section 4 .

\section{Models and Methods}

\subsection{Models}

[6] The GCM used in this study is GFDL AM2 version am2p14, an atmospheric GCM developed at the Geophysical Fluid Dynamics Lab (GFDL) [The GFDL Global Atmospheric Model Development Team, 2004]. This version employs a finite-volume dynamical core [ $\operatorname{Lin}, 2004]$ with a $2.5^{\circ}$ longitude by $2^{\circ}$ latitude resolution. It has 24 vertical levels with two layers in the TTL, one centered at $158 \mathrm{hPa}$ and the other 
at $100 \mathrm{hPa}$. The cumulus parameterization is a relaxed Arakawa-Schubert scheme. Large-scale cloud ice water content (IWC) and cloud fraction are treated as prognostic variables. Ramaswamy et al. [2006] showed that when both anthropogenic and natural factors are included, AM2 is capable of simulating the satellite-observed spatial and temporal structures of cooling in the lower stratosphere for the last two decades. The trajectory model employed in this study is the latest version of HYSPLIT (Hybrid Single-Particle Lagrangian Integrated Trajectory) model, which was developed and validated by the NOAA Air Resources Laboratory [Draxler, 1996a, 1996b].

\subsection{Methods}

[7] We run the AM2 model with observed SST starting from 2000 and, as a case study, analyze the four-month model output of April-July 2003. 3-hourly instantaneous fields of simulated winds, temperatures, cloud properties, and radiative heating rates are archived from the simulation. At 1200 UTC of each day in July 2003 (hereafter referred as "Hour zero" or H0), air parcels are initialized at $70 \mathrm{mb}$ in the inner tropics $\left(15^{\circ} \mathrm{S}-15^{\circ} \mathrm{N}\right)$ with 1-degree separation in latitude and 1.25-degree separation in longitude. As by Bonazzola and Haynes [2004], a 90-day back trajectory is then calculated for each air parcel based on the archived 3-hourly wind fields.

[8] To describe the cloud influence on these trajectories, we define the TTL-cloud residence time for each trajectory as

$$
t_{c l d}=\sum_{i=-N}^{0} F_{c l d}[\lambda(i), \varphi(i), p(i)] \Delta t
$$

where $i$ is hours before H0, and the air parcel ascends through the base of TTL $(150 \mathrm{mb})$ at hour $-N . \lambda(i), \varphi(i)$, and $p(i)$ refer to the latitude, longitude, and pressure level of the air parcel at hour $i$, respectively. $\mathrm{F}_{\text {cld }}$ is the local cloud fraction, which is archived from the AM2 simulation. $\Delta \mathrm{t}$ is the time interval, which equals one hour since the back trajectory calculation is carried out on an hourly basis. $t_{c l d}$ represents how long an air parcel stays inside clouds while it is in the TTL. $t_{c l d}=0$ indicates an entirely cloud-free trajectory through the TTL and $t_{c l d}=\mathrm{N}$ indicates a trajectory that resides entirely in the TTL clouds.

[9] To represent the cloud structure underneath the TTL cirrus, we categorize three different cloudy scenes (CS) based on the model's UT (three layers between $420 \mathrm{mb}$ and $191 \mathrm{mb}$ ) cloud properties. Scene 1 (hereafter, CS1) corresponds to optically thick clouds in the UT, where cloud fraction in the UT ( $\mathrm{f}_{\text {cld UT }}$ ) is larger than 0.8 and the $11 \mu \mathrm{m}$ optical depth of the UT cloud ( $\left.\tau_{\text {cld UT }}\right)$ is larger than 3 . Scene 2 (hereafter, CS2) corresponds to minimal presence of clouds in the UT, with $\mathrm{f}_{\text {cld_UT }}<0.05$ and $\tau_{\text {cld_UT }}<0.1$. All other scenes are categorized Scene 3 (CS3).

\section{Results}

3.1. Simulated April-July Mean Properties in the TTL

[10] Figures $1 \mathrm{a}$ and $1 \mathrm{~b}$ show the simulated mean IWC and cloud fraction in the TTL from April-July 2003. The regional maxima of IWC over the Indian subcontinent and western Pacific warm pool can be seen in both layers of the
AM2 TTL while the IWC maximum over the tropical eastern Pacific is only evident in the lower layer. All three regional maxima in the TTL are also present in the UT, indicating their connection with the ITCZ and summer Indian monsoon as simulated in the model. The regional maxima of mean cloud fraction in the TTL (Figure $1 \mathrm{~b}$ ) are about $50-70 \%$. They correspond to the maxima in IWC but encompass larger areas, likely due to the horizontal advection of cirrus clouds.

[11] As a measure of the temporal variability of clouds, the standard deviation of the 3-hourly simulated cloud fraction is shown in Figure 1c. Regions with large mean cloud fraction tend to have large standard deviations as well. Some regions have minimal mean cloud fraction $(<3 \%)$ but substantial standard deviation $(\sim 5-10 \%)$ e.g., southernhemisphere subtropical eastern Pacific $\left(135-90^{\circ} \mathrm{W}\right.$ and $\left.10-15^{\circ} \mathrm{S}\right)$. An examination of the time series of cloud fraction vertical profiles reveals that, although the TTL of such regions is cloud-free most of the time, occasionally insitu cirrus forms in large amounts and lasts for several hours to one day. Such sporadic events account for the measurable standard deviation in spite of nearly zero mean cloud fraction.

[12] The in-cloud radiative heating rates (i.e., the difference between all-sky and clear-sky radiative heating rates in the TTL), which measures the radiative contribution of clouds to the diabatic heating, is shown in Figure 1d. For regions with maximum IWC, the net radiative effect of TTL cirrus is to heat the TTL by $0.3-0.5 \mathrm{~K} /$ day. The cloud heating effect over the tropical eastern Pacific is weaker than those over the other two IWC maxima because cirrus there is confined to the base of the TTL, rather than distributed over both TTL layers in the model. The mean vertical velocity in the TTL is plotted in Figure 1e. The regions with strongest upward velocity in Figure 1e coincide with those with largest IWC in Figure $1 \mathrm{a}$.

\subsection{Back-Trajectory Results}

[13] Among trajectories that we calculated, 25.2\% enter the base of the TTL $(150 \mathrm{mb})$ within 90 days before H0, $74.6 \%$ enter the TTL beyond 90 days, and $0.2 \%$ originate from the stratosphere above. The last group of the trajectories is excluded in the following analysis since it is irrelevant to the objective of this study and its fractional contribution is negligible.

\subsubsection{Trajectories Across the TTL Within 90 Days}

[14] Histograms counting times of entry to the TTL and TTL cloud residence times of the trajectories are shown in Figure 2. The distribution of cloud residence time is positively skewed with a sharp peak around 0.86 days. The maximum amount of time spent inside the TTL clouds by a trajectory is 13.27 days. The histogram of entry time, on the other hand, generally increases monotonically when the entry time is further away from $\mathrm{H} 0$. As a result, the histogram of the fraction of time that an air parcel stays inside the clouds in the TTL is also positively skewed with a sharp peak around $4.1 \%$. These histograms indicate that the air parcels generally stay in the clear-sky portion of the TTL. This is consistent with the relatively confined cloudy regions and the vast clear regions as shown in Figure 1a.

[15] Figure 3 shows composites of mean vertical velocity $(\bar{\omega})$ and mean radiative heating rate $\left(\bar{q}_{\text {rad }}\right)$ with respect to 

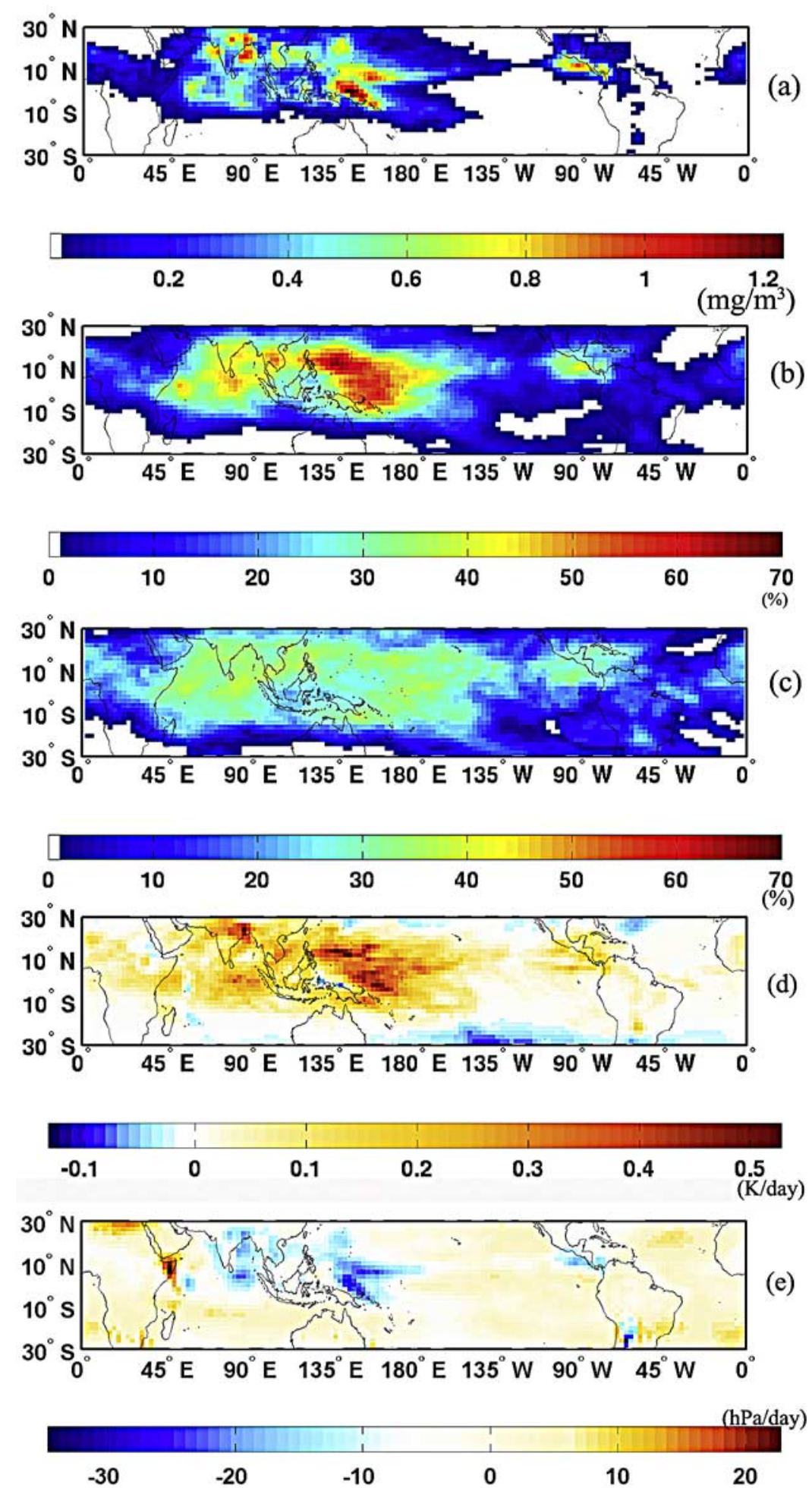

Figure 1. (a) The simulated mean ice water concentration $\left(\mathrm{mg} \mathrm{m}^{-3}\right)$ in the TTL over April-July 2003. (b) Same as Figure 1a except for the TTL cloud fraction (\%). (c) Standard deviations of the simulated 3-hourly cloud fraction (\%) in the TTL over the same period. (d) Same as Figure 1a except for the difference between all-sky and clear-sky radiative heating rates (K/day) in the TTL. (e) Same as Figure 1a except for the mean vertical velocity (hPa/day) in the TTL.

cloud residence time and fractional overlapping with thick clouds in the UT (CS1 defined in section 2.2). As expected, $\bar{q}_{\text {rad }}$ is small when the occurrence of CS1 is frequent, since the resulting thermal contrast between TTL cirrus and underlying clouds are small. $\bar{q}_{\text {rad }}$ increases as the occurrence of CS1 decreases(Figure 3b). If the ascending rates are primarily controlled by $\bar{q}_{\text {rad }}$, the strongest $\bar{\omega}$ would coincide with regime of the largest $\bar{q}_{\text {rad }}$. However, as shown in 


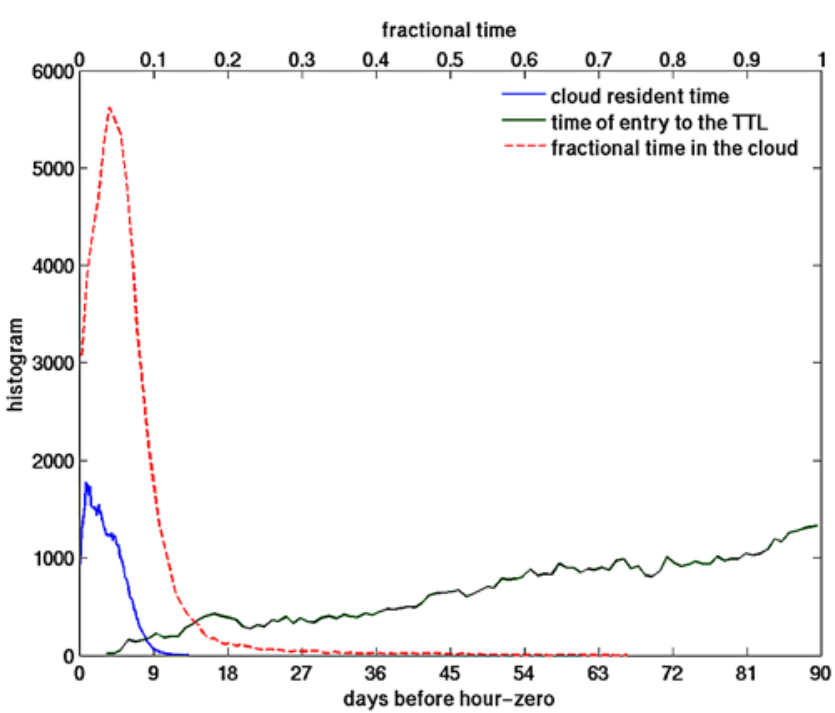

Figure 2. Histograms of TTL cloud residence time (blue line), time of entry to the TTL (green line), and the fractional time that an air parcel stays in the TTL cloud along the trajectory (dashed red line). 100 bins from minimal to maximum values are used for each histogram.

Figure $3 \mathrm{a}$, the strongest composite vertical velocity corresponds to a regime with relatively weak $\bar{q}_{r a d}$, cloud residence time around $0.5-2$ days (encompassing the peak of the histogram of cloud residence time in Figure 2), and 30$60 \%$ time of overlapping with thick UT clouds. These conditions tend to be related to strong upward motion through the entire tropospheric column, usually associated with deep convection. Thus, in the AM2, the fastest TST transport (i.e. the strongest $\bar{\omega}$ ) is not directly driven by the cloud radiative effect, but by dynamics. To the left of this regime in Figure 3a, when cloud residence time decreases, the trajectories spend less time inside the TTL clouds and more time in clear sky, resulting in weaker ascent. To its right, as time spent in the TTL clouds increase and overlap with thick UT clouds decreases, $\bar{q}_{\text {rad }}$ and upward velocity (more negative $\bar{\omega}$ ) both increase. This indicates that TST in such a regime is less influenced by dynamics and more influenced by the radiative effect of the TTL clouds.

[16] Although the strongest $\bar{\omega}$ (between -4.5 and $-3.5 \mathrm{hPa} /$ day) can be attributed mainly to dynamic causes, the number of trajectories (contour lines in Figure $3 \mathrm{a}$ or $3 \mathrm{~b}$ ) belonging to this regime is one order of magnitude smaller than those belonging to moderate $\bar{\omega}$ regime (between -2.5 and $-3.5 \mathrm{hPa} /$ day), where the cirrus radiative effect is significant. The upward motion due to clear-sky radiative effect $\left(\omega_{\text {rad_clr }}\right)$ in the TTL of AM2 can be estimated by assuming balance between clear-sky net radiative heating and adiabatic cooling associated with ascending motion. Using the AM2 mean inner tropical profiles over this period (April-July 2003), such estimation leads to a $\omega_{\text {rad_clr }}$ of $-0.18 \mathrm{hPa} /$ day, one order of magnitude smaller than the aforementioned moderate $\bar{\omega}$. Therefore, the cirrus radiative effect has a non-negligible effect on tropical-mean TST transport rates, as postulated by $\mathrm{C} 05$ and $\mathrm{C} 06$.

\subsubsection{Composites of All Relevant Trajectories as Well as Eulerian Grid-Boxes}

[17] In this subsection, trajectories across the TTL within the 90-day period as well as those across the TTL beyond 90 days are analyzed together in the following way: for every hour a trajectory stays in the TTL, the scene type is identified as clear-sky, CS1, CS2, or CS3; then the vertical velocity $(\bar{\omega})$, net radiative heating rate $\left(\bar{q}_{\text {rad }}\right)$ and occurrence frequency are composited for each scene type. Note that $\bar{\omega}$ and $\bar{q}_{\text {rad }}$ here are averages of instantaneous output over the same scene types, not averages along the entire trajectory. The results are summarized in Table 1. As above, the strongest $\bar{\omega}$ occurs in CS1 (overlapping with thick UT clouds) even though its $\bar{q}_{\text {rad }}$ is not as large as that in CS2. The probability of encountering CS1 is smaller than that of other cloudy scenes. Including trajectories with entry time beyond 90 days greatly increases the occurrence of clearsky scenes $(>90 \%) . \bar{\omega}$ of the clear-sky scene is one order of magnitude smaller than the $\bar{\omega}$ of the cloudy scenes, consistent with the results in the previous section, and $\mathrm{C} 05 / \mathrm{C} 06$.
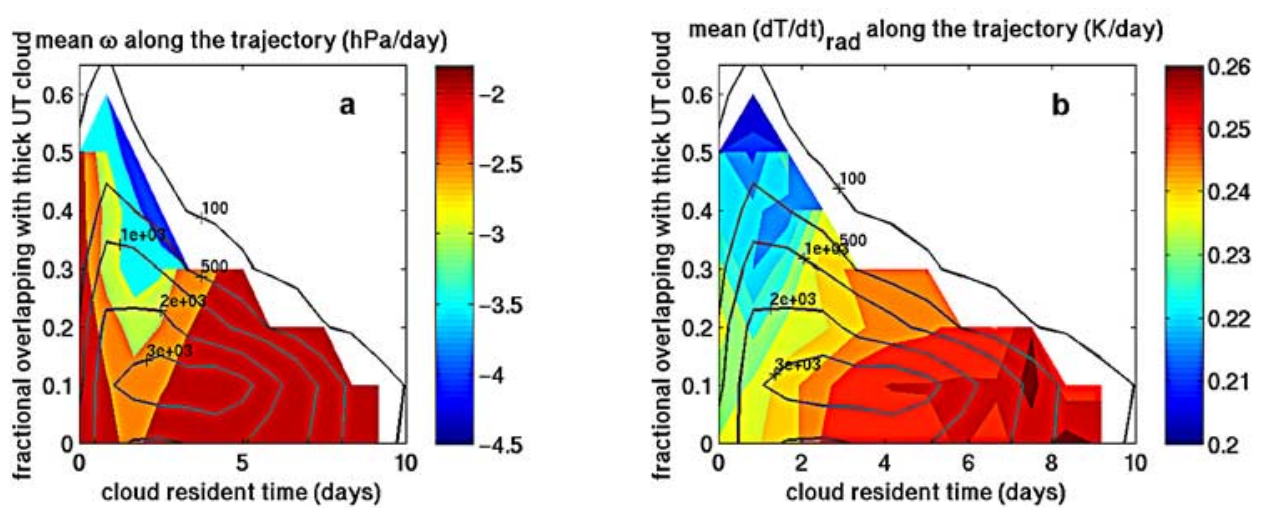

Figure 3. (a) The composite of mean vertical velocity along the trajectories with respect to cloud residence time (20 equally spaced bins) and the frequency of encountering CS1 along the trajectory (10 equally spaced bins). The contour line shows the number of trajectories in each bin. For the consideration of statistical robustness, bins with less than 100 trajectories are discarded. (b) Same as Figure 3a except for the composite of mean radiative heating rate along the trajectories. 
Table 1. Statistics (mean \pm standard deviation) of AM2-Simualted Vertical Velocity and Net Radiative Heating Rate in the TTL for Different Scene Types ${ }^{\mathrm{a}}$

\begin{tabular}{|c|c|c|c|c|c|c|}
\hline & \multicolumn{3}{|c|}{ Trajectory-Based Statistics } & \multicolumn{3}{|c|}{ 6-Hourly Eulerian Field Statistics } \\
\hline & $\omega_{\text {TTL }}(\mathrm{hPa} /$ day $)$ & $\left(\mathrm{dT}_{\mathrm{rad}} / \mathrm{dt}\right)_{\mathrm{TTL}}(\mathrm{k} /$ day $)$ & Occurrences & $\omega_{\mathrm{TTL}}(\mathrm{hPa} /$ day $)$ & $\left(\mathrm{dT}_{\mathrm{rad}} / \mathrm{dt}\right)_{\mathrm{TTL}}(\mathrm{k} / \mathrm{day})$ & Occurrences \\
\hline Clear-sky & $-0.26 \pm 5.65$ & $0.17 \pm 0.37$ & $92.9 \%$ & $2.14 \pm 8.31$ & $0.15 \pm 0.23$ & $46.6 \%$ \\
\hline Cld scene1 & $-5.93 \pm 22.74$ & $0.14 \pm 0.50$ & $0.7 \%$ & $-10.58 \pm 22.78$ & $-0.06 \pm 0.52$ & $6.7 \%$ \\
\hline Cld scene 2 & $-3.02 \pm 6.24$ & $0.52 \pm 0.32$ & $1.6 \%$ & $1.08 \pm 8.31$ & $0.31 \pm 0.33$ & $6.2 \%$ \\
\hline Cld scene 3 & $-5.02 \pm 10.03$ & $0.44 \pm 0.36$ & $4.8 \%$ & $-2.97 \pm 13.79$ & $0.32 \pm 0.41$ & $40.5 \%$ \\
\hline
\end{tabular}

${ }^{\text {a }}$ Statistics are mean \pm standard deviation. The occurrence of each scene type is shown in percentage.

[18] The same scene type classification and statistical analysis can be applied to the 6-hourly instantaneous gridded AM2 output. The results are also presented in Table 1 . Statistics from an Eulerian point of view will differ from those based on parcel trajectories unless all TTL locations have an equal opportunity to be visited by an ascending air parcel. For example, TTL regions featuring with frequent downward motion (or radiative cooling) will be included in the Eulerian analysis but will be less preferred in our Lagrangian trajectory analysis. As a result, $\bar{q}_{\text {rad }}$ from the Eulerian analysis is smaller than its trajectory analysis counterparts for all scene types (Table 1), as is upward velocity $\bar{\omega}$ for clear-sky scene, CS2, and CS3. The occurrence of CS1 is 10 times larger than that in the trajectory analysis, and the CS1 composite $\bar{\omega}$ is much stronger. In composites based on parcel trajectories, horizontal advection driven by strong horizontal winds moves air parcels away from the strongest ascending regions, causing a weaker $\bar{\omega}$.

\section{Conclusion and Discussion}

[19] Analyses of the simulated TTL cirrus radiative effects on ascending motion in the GFDL AM2 are presented from both Eulerian and Lagrangian point of views. The results indicate that the hypotheses by $\mathrm{C} 05$ and $\mathrm{C} 06$ are largely valid in this GCM (i.e., cirrus radiative heating can contribute substantially to the overall TST). This relation does not generalize to locations where TTL cirrus overlays with thick UT cloud decks. In such scenes, dynamic-driven ascent in the model is stronger than radiative-driven ascent; however the occurrence frequency of radiative-driven ascent is larger by orders of magnitude.

[20] It has been noted that current GCMs have limitations in representing the tropical TST, especially given the coarse resolutions in the TTL. Two lines of research are advancing to resolve this limitation. First, new model development is underway to better resolve the TTL and represent tropical TST (e.g. the next generation of GFDL model has 4 layers between $70 \mathrm{mb}$ and $170 \mathrm{mb}$, double the current resolution in the AM2). In addition, global-scale observations of TTL clouds together with underlying cloud properties have been tremendously improved by several A-train instruments, chiefly CALIPSO, CloudSat, MLS, and HIRDLS. The asynoptic sampling (around 1:30 am/pm local time) of these A-Train satellite measurements limits the use of these data in a traditional Eulerian way. The scene-type composites along Lagrangian trajectories used in this study, on the other hand, can be extended for use with such A-Train satellite datasets. The accumulated observational statistics can then be compared to the output from current or new GCM simulations.

[21] Acknowledgments. We thank J. Jiang, Z. Luo, and W. Read for valuable discussions. We wish to thank two anonymous reviewers for valuable suggestions on improving the clarity of the presentation. We are thankful to V. Ramaswamy and NOAA GFDL for generously providing the computing resources for the AM2 simulation. This research is partly supported by a JPL DRDF grant (DRDF-632506) awarded to the two authors. Hui Su's work was carried out at the Jet Propulsion Laboratory, California Institute of Technology, under contract with NASA.

\section{References}

Andrews, A. E., K. A. Boering, B. C. Daube, S. C. Wofsy, E. J. Hintsa, E. M. Weinstock, and T. P. Bui (1999), Empirical age spectra for the lower tropical stratosphere from in situ observations of $\mathrm{CO}_{2}$ : Implications for stratospheric transport, J. Geophys. Res., 104(D21), 26,581-26,596, doi:10.1029/1999JD900150.

Boering, K. A., B. C. Daube Jr., S. C. Wofsy, M. Loewenstein, J. R. Podolske, and E. R. Keim (1994), Tracer-tracer relationships and lower stratospheric dynamics: $\mathrm{CO}_{2}$ and $\mathrm{N}_{2} \mathrm{O}$ correlations during SPADE, Geophys. Res. Lett., 21(23), 2567-2570, doi:10.1029/94GL01985.

Bonazzola, M., and P. H. Haynes (2004), A trajectory-based study of the tropical tropopause region, J. Geophys. Res., 109, D20112, doi:10.1029/ 2003JD004356.

Brewer, A. W. (1949), Evidence for a world circulation provided by the measurements of helium and water vapor distribution in the stratosphere, Q. J. R. Meteorol. Soc., 75, 351-363.

Corti, T., B. P. Luo, T. Peter, H. Vomel, and Q. Fu (2005), Mean radiative energy balance and vertical mass fluxes in the equatorial upper troposphere and lower stratosphere, Geophys. Res. Lett., 32, L06802, doi:10.1029/2004GL021889.

Corti, T., B. P. Luo, Q. Fu, H. Vomel, and T. Peter (2006), The impact of cirrus clouds on tropical troposphere to stratosphere transport, Atmos. Chem. Phys., 6, 2539-2547.

Dessler, A. E., and K. Minschwaner (2007), An analysis of the regulation of tropical tropospheric water vapor, J. Geophys. Res., 112, D10120, doi:10.1029/2006JD007683.

Dessler, A. E., and S. C. Sherwood (2000), Simulations of tropical upper tropospheric humidity, J. Geophys. Res., 105(D15), 20,155-20,163.

Draxler, R. R. (1996a), Boundary layer isentropic and kinematic trajectories during the August 1993 North Atlantic regional experiment intensive, J. Geophys. Res., 101(D22), 29,255-29,268.

Draxler, R. R. (1996b), Trajectory optimization for balloon flight planning, Weather Forecasting, 11,111-114.

Fueglistaler, S., H. Wernli, and T. Peter (2004), Tropical troposphere-tostratosphere transport inferred from trajectory calculations, J. Geophys. Res., 109, D03108, doi:10.1029/2003JD004069.

Gettelman, A., P. M. de F. Forster, M. Fujiwara, Q. Fu, H. Vömel, L. K. Gohar, C. Johanson, and M. Ammerman (2004), Radiation balance of the tropical tropopause layer, J. Geophys. Res., 109, D07103, doi:10.1029/ 2003JD004190

Hartmann, D. L., J. R. Holton, and Q. Fu (2001), The heat balance of the tropical tropopause, cirrus, and stratospheric dehydration, Geophys. Res. Lett., 28(10), 1969-1972.

Holton, J. R., and A. Gettelman (2001), Horizontal transport and the dehydration of the stratosphere, Geophys. Res. Lett., 28(14), 2799-2802.

Holton, J. R., P. H. Haynes, M. E. McIntyre, A. R. Douglass, R. B. Rood, and L. Pfister (1995), Stratosphere-troposphere exchange, Rev. Geophys., 33(4), 403-439. 
Jensen, E., and L. Pfister (2004), Transport and freeze-drying in the tropical tropopause layer, J. Geophys. Res., 109, D02207, doi:10.1029/ 2003JD004022.

Lin, S. J. (2004), A "vertically Lagrangian" finite-volume dynamical core for global models, Mon. Weather Rev., 132, 2293-2307.

Ramaswamy, V., and V. Ramanathan (1989), Solar absorption by cirrus clouds and the maintenance of the tropical upper troposphere thermal structure, J. Atmos. Sci., 46, 2293-2310.

Ramaswamy, V., et al. (2006), Anthropogenic and natural influences in the evolution of lower stratospheric cooling, Science, 311, 1138-1141.

Sherwood, S. C., and A. E. Dessler (2001), A model for transport across the tropical tropopause, J. Atmos. Sci., 58, 765-779.
Sherwood, S. C., and A. E. Dessler (2003), Convective mixing near the tropical tropopause: Insights from seasonal variations, J. Atmos. Sci., 60, $2674-2685$

The GFDL Global Atmospheric Model Development Team (2004), The new GFDL global atmosphere and land model AM2-LM2: Evaluation with prescribed SST simulations, J. Clim., 17, 4641-4673.

X. Huang, Department of Atmospheric, Oceanic, and Space Sciences, University of Michigan, Ann Arbor, MI 48109-2143, USA. (xianglei@ umich.edu)

H. Su, Jet Propulsion Laboratory, California Institute of Technology, 4800 Oak Grove Drive, Pasadena, CA 91109, USA. 